\title{
The Future of Swelling Elastomers: An Elastomer Manufacturer's View of Swelling Elastomer Developments and Market Trends
}

\author{
R Seyger, S Resink, H Harms and R Hibberd* \\ RUMA Products BV, Zeppelinstraat 5, 7903 BR Hoogeveen, The Netherlands
}

Received 24 August 2012 ; accepted 28 November 2012

\begin{abstract}
Swelling elastomers have gained acceptance as very effective products for creating sealing in various industries, including those creating energy from fossil fuels and geothermal resources. This paper outlines the research and development work being conducted not only in the application of these elastomers but also in the development work required to create new generations of elastomers. It touches on fundamental research into the mechanics of swelling with the intent to create a better and more predictable sealing as well as more advanced elastomers. It lifts the veil on the direction of work being done on new elastomers being developed in order to enable a better control of swelling. By doing so, the research is opening up field of applications for new equipment designs and mechanical possibilities in the future. Additionally, it addresses the need for a better and more in-depth dialogue between both chemical and mechanical engineers, and the elastomer companies and their customers on the potential that both swelling and non-swelling elastomers can offer to the industry as a whole.
\end{abstract}

Keywords: Swelling elastomers, Sealing, Water-swell, Oil-swell

$$
\begin{aligned}
& \text { مستقبل اللدائن المتورمة: رؤية مصنعي اللدائن المتورمة حول تطورات واتجاهات } \\
& \text { السوق لهذا النوع من المواد } \\
& \text { روجر سيهجير، شورد ريزينك، هيرمان هارمز، روجر هايبرد" المواد مهن }
\end{aligned}
$$

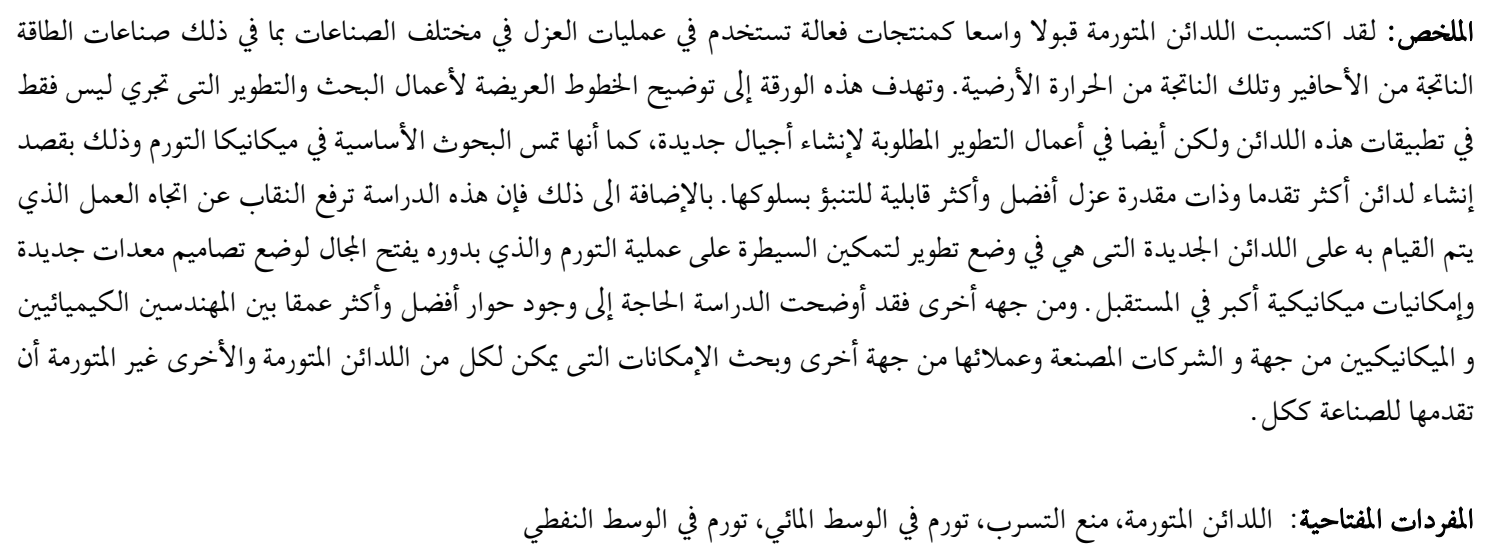

\section{Introduction}

All elastomers can be and are affected by the different chemical substances with which they come into contact. The effects of this contact can vary; however, one of the most common effects is that of the elas-

*Corresponding author's e-mail: r.hibberd@oblique.eu tomer physically swelling. Upon contact with fluids such as oil and water and, under the correct conditions, elastomers will almost always swell. However, depending upon the base elastomer, this can vary from an almost negligible amount to a quite large volume 
swell of $100-150 \%$. The degree of swell is dependent upon the fluid conditions and the elastomer type and design. The swell is effectively a result of oil or water interacting with one or more of the chemical ingredients used in the production of the elastomer. These ingredients were originally intended to enhance or influence one or another of the elastomer's mechanical properties used during the manufacturing process. Over the years, a huge amount of work and research has been conducted to lower or eliminate the degree of swell while maintaining the desired mechanical properties. This swell is regarded and experienced as an undesirable effect. However, because elastomers can swell in very selective fluids and this swell can be to different degrees, it sometimes has been enhanced so as in some industries or applications to be considered a potentially useable characteristic. For years, this swell has been utilized in mining and civil engineering to create seals, and for sealing against fluid ingress (Pal et al. 2010).

Whilst these so-called swelling elastomers have been around so long and are used in various forms by some major industries, their general acceptance and use in the oil and gas fields has been relatively recent. After initially proving their worth, they have eventually gained acceptance in the oil patch, and a whole new series of applications have been created for them which center around their unique nature. This has opened up new economic production opportunities and created new use areas to operators. Probably the most widely known and talked about of these areas has been the step change they have brought as "fracturing" seals to the shale oil and gas industries (Anjum 2010).

Here the available conventional mechanical techniques to open up the tight gas formations had technical limitations and in turn were economically restricting the production of vast reserves of so called "tight gas". The availability of swelling seals, where these could just be run and left to swell, rather than the conventional mechanical packers that needed to be operated via intervention from the surface, enabled simple quick and easy multilateral design and installation for reservoir fracturing to occur. This resulted in the freeing up of relatively cheap and substantial reserves of hydrocarbon gas and, in some newer shale oil fields, cheap and effective production. A new worldwide resurgence in interest in the long known of but economically unviable reserves has been created. In the key use area of the USA, this has led to a glut in gas and with the resulting low price has come a radical change in other industries and their use of gas in general. In the USA, gas is now replacing coal in power generation and is being used as feed stock for many existing products (Young 2011).

However, in spite of the acceptance by the oil and gas industry as a whole, little real new development has occurred to swelling elastomers themselves. Rather, the work has centered only on the technical application of the elastomers. Most of the elastomer changes, where they have happened, are just small refinements to the base elastomers' performance. Basically, the elastomers have not substantially changed in the last 5 to 10 years (Wack 2011). As designers and manufacturers of elastomers for use in products, we are involved not just with the present generations of swelling compounds, but we are also doing both fundamental and applied research, and development into the next generation and beyond.

\section{Temperature Ranges, Low and High}

While there have been a multitude of different swelling seals that will swell in various different fluids, and some very specific ones that swell only in acids or alkaline fluids, this study will look at the work done in swelling in oil and/or water as this is the major market that is of interest to most people at the moment.

So where are we as a company going with the development of the next generations of elastomers? The market for swelling elastomers has moved from simple mining and civil engineering applications through to the first few oil, water, and hybrid swelling elastomer seals trialed and introduced into operations by national and international oil companies. This introduction has been into a market that has been predicted to approach or, in some studies, exceed over a third of a million seal items per year by 2014-15. This reflects quite a huge use increase, occurring in a little less than a 10-year time frame. It is difficult, however, to think that this is just a drop in the bucket when considering what is actually coming.

The demand is out there and is growing for a new generation of elastomers designed to deal with more extreme environments and conditions, with demand for both higher and lower temperature ranges than have been the application norm to date. The first of these demands is for a much lower temperature application. Most existing swelling elastomers perform reasonably well in temperatures down to about $40-50^{\circ}$ Celsius but, at this low temperature, the rate of swelling slows substantially. At any temperature much lower than this, standard seals and especially oil swell elastomers start to become a problem as most oils at low temperatures are quiet viscous and the diffusion gradient causing swell is quite low. When coupled with the hardness of the elastomer at lower temperatures, the result is a slow swell coupled with a low level of absolute swelling. While oil swell is proving more of a problem at low temperatures, water swell fares slightly better with reasonable swelling down to $20-25^{\circ} \mathrm{C}$ as long as the fluid salinity is not too high. However, to be able to improve performance, a deep 
understanding of elastomer swelling is required, especially as all of these improvements are taking place in a continually price-orientated market. Elastomer swelling and mechanical performance on the one side has to be coupled with improved manufacturing performance of the elastomer on the other. This enables elastomers to have not only improved technical performance but also to be commercially attractive to users.

As the operating envelopes move both up and down, the quality of the ingredients as well as the quality of the manufacturing of the elastomers demand improved ingredient specifications as well as a far tighter quality control in manufacturing. If these standards are not met, the performance envelope for the elastomer becomes too variable. Additionally, chemical ingredients are being rationed by manufacturers and suppliers, resulting in a frequent need to find and test comparable products from new or different sources to ensure quality is maintained or improved upon.

\section{How Do They Work?}

\subsection{Basic Principles}

We are aware that many engineers are not comfortable in their understanding of the mechanics of how swelling elastomers work. They know that they do swell, but the explanation as to why is often one that a chemical engineer would understand but is not easily grasped by a mechanical engineer. Conversely, the swelling phenomenon is often presented by supplier sales staff as a form of magic that is performed. This notion can be advanced simply because many do not understand the process themselves. This lack of understanding is partially due to scientists' different disciplines and backgrounds, but can also be explained by the fact that some terms in chemistry differ from those commonly used in mechanical engineering. Whether it is an oil or water swell or, for that matter, any other type of fluid-motivated swelling, it is the result of an entropy difference between the elastomer and the environment in which it is being used. This energy disparity manifests itself in an effort to achieve an energy balance by creating a diffusion gradient between the elastomer and the fluid around it. For details of Fick's first and second laws of diffusion, please see the appendix (Wikipedia 2012).

Figure 1 shows how by modeling the various physical mechanical parameters of the elastomer and chemical parameters of the elastomer in the motive fluid, it is possible to predict the anticipated performance of swelling elastomers prior to actually mixing the ingredients. This then enables rapid prototype modeling on paper to precede the compound design prior to making and testing in the lab, thus speeding up and optimizing the compounding process while reducing development costs and unnecessary research time. In this way, the work centers around confirming the desired characteristics and those results are being achieved.

\subsection{Oil Swell Elastomers}

In oil swelling, one or more of the distillate fractions in the oil has an affinity for the base elastomer and starts via the diffusion gradient to dissolve the hydrocarbon chain that forms a part of the elastomer. This results in an oil fraction being pulled into the elastomer and a dissolving and breaking down of the long chains that form the elastomer. Because the motive fluid (oil) is incompressible, as is the elastomer, this pulling in of oil fractions results in a substantial force within the elastomer. This elastomer mix is then stretched much like blowing up a balloon where the air in a balloon is being replaced by a soup of hydrocarbons made up of the oil fraction and dissolving elastomer. The resulting effect is a physical swelling of the elastomer. (Note that in water swell the elastomer is not directly taking part in the process so is not itself changing in volume unless the base elastomer type is an oil swelling one as used in multifunctional hybrid elastomers). The term "base elastomer" refers to the elastomer matrix that is used and with it the different elastomers and elastomer mixtures that can be used such as acrylonitrile-butadiene rubber (NBR), hydrogenated nitrile butadiene rubber (HNBR), ethylene propylene diene monomer rubber (EPDM), silicones, etc.

Figure 2 shows how a change in elastomer characteristics (different elastomer types 1 to 5), together with an alternative base test oil such as an intermediate restorative material (IRM) or diesel, or the same elastomer and fluids but with a different temperature alters the swelling characteristics achieved in oil swelling elastomers. This makes accurate swell performance predictions for oil swell elastomers very difficult without actually lab testing the specific motive fluids.

\subsection{Water Swell Elastomers}

Water swell works in the same way as oil swell, (ie. a diffusion gradient being created, except that the water now has an affinity via a diffusion gradient for one or more of the ingredients in the elastomer). However, it does differ in that the base elastomer is not affected by the motive water as is the case with oil swelling. There is a widely held belief that the swelling is due to specific ingredients being added to create the swell; however, this is not necessarily the case. The water's affinity can be for the plasticizers used, the vulcanization enhancers or even one of the many ingredients used to enable the elastomer to flow easily during the molding or production processes. In 


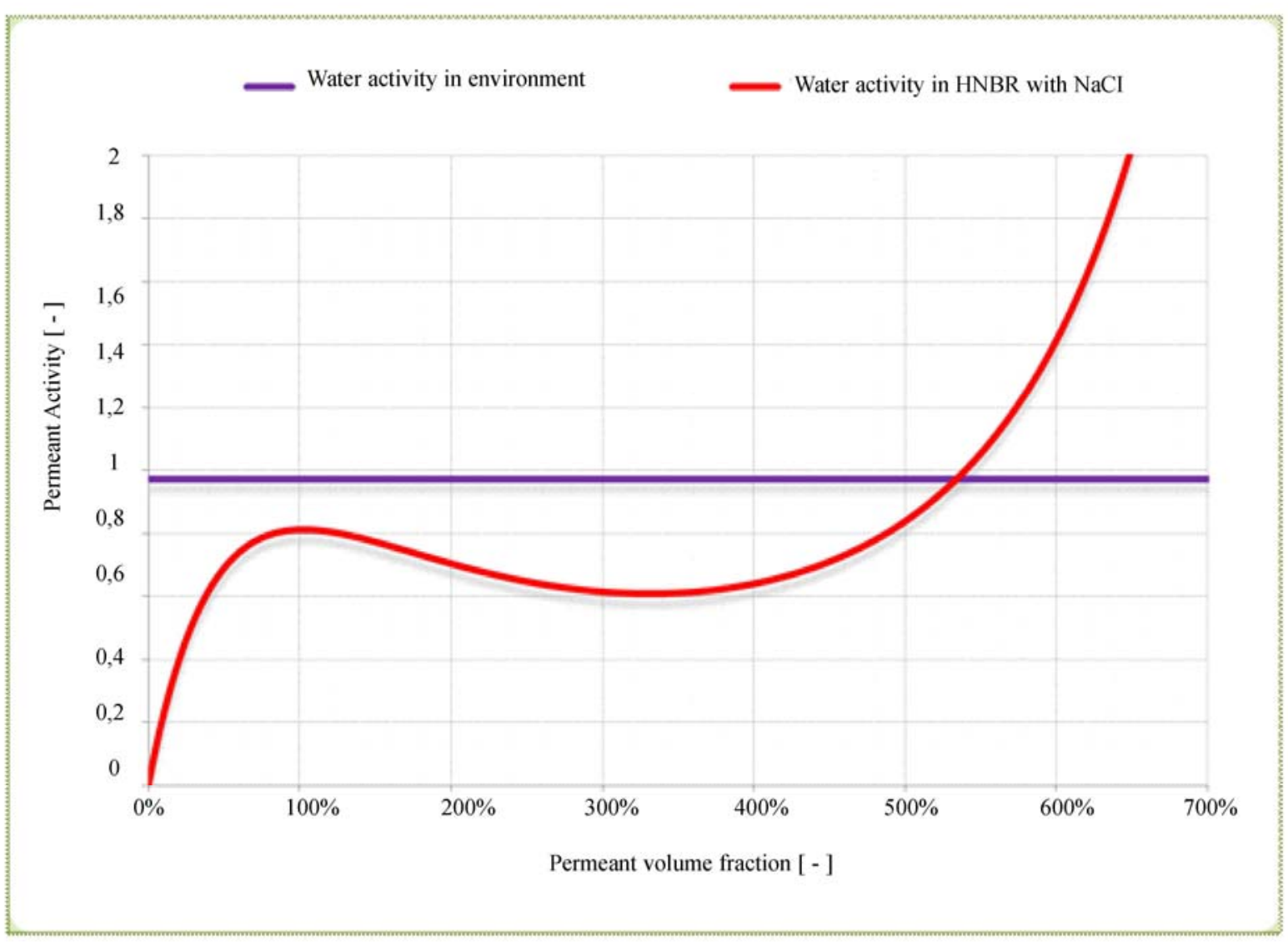

Figure 1. Modeling for physical and chemical parameters of a swelling elastomer

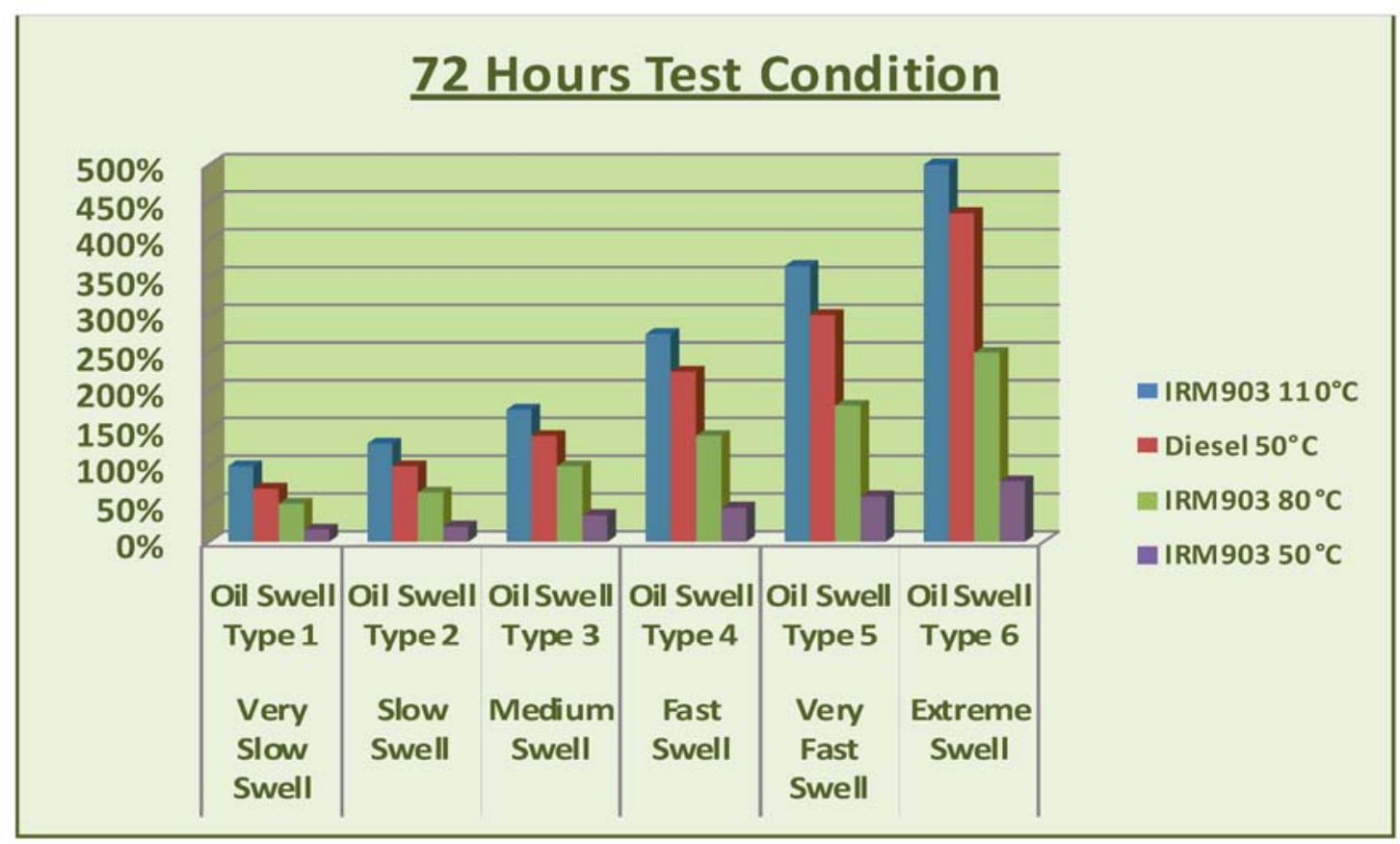

Figure 2. Swelling performance of five different elastomers in different mediums

some instances, ingredients such as salt super absorbent polymers (SAPs) that have been added are not to aid manufacture but to enhance, create, or initi- ate swelling. However, what is the key, and is something different from oil swelling is that the base elastomer does not actually take part in the swelling process other than to form the "balloon" that accom- 


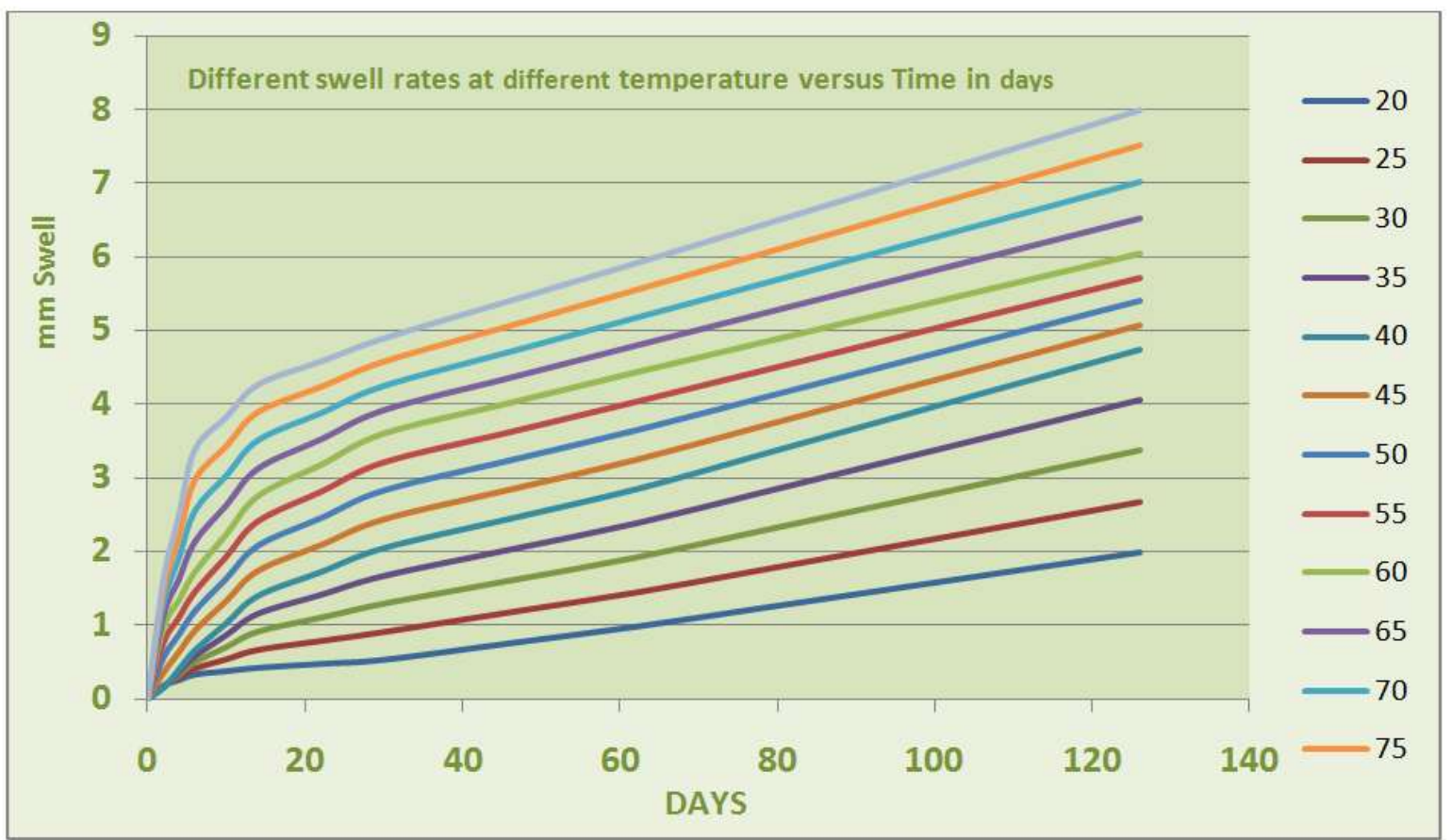

Figure 3. Amount of swelling for one elastomer against swelling time under the same medium at different temperatures

modates the fluid that is actually creating the swell. Please see the appendix for a simple explanation about the working of SAPs (M2 Polymer Technologies 2012).

Because the swell is a physical interaction between the motive fluid and the base elastomer, the design of the swelling elastomer base is critical. If ingredients are being added in water swelling applications specifically to create the swell, a number of things become very important. The more ingredients that are added, the less elastomer per unit volume remains. It goes without saying that the elastomer imparts strength to the product. This means that to maintain the strength of the whole, the elastomer remaining has to have increased strength (more cross linking). The higher the crosslink density the more rigid the elastomer; the more it resists the load created by the fluid, the less the swell becomes. This is a very complex inter-relationship with a fine balance between swell performance and mechanical integrity for both sealing and pressure containment. The internal pressure being generated by reducing the diffusion gradient through swelling is what is being utilized to create the basis for the sealing force.

Ingredient quality, mixing order, and vulcanization quality and control are vital to enable a swelling product to be created with a well-designed and consistent sealing performance envelope. The effects low and high temperatures have on diffusion gradients as well as elastomer mechanical performance are crucial for the creation of commercially technically viable prod- ucts with a working life that is comparable to so called non-swelling elastomers in mechanical packers. Poorly designed or tested seals are easy to achieve but can be a very disappointing product in terms of longterm mechanical integrity and performance.

While conventional mechanical packers are created by mechanical engineers and utilize one of the worst mechanical characteristics of elastomers, swelling elastomer seals are radically different in that they are created by chemical engineers using chemical engineering and utilize its best mechanical characteristics. This engineering change is something very new to the oil field.

Figure 3 illustrates the different absolute swell that can occur in an elastomer in a fixed salinity with a change in fluid temperatures versus time. What is apparent is the very steep rapid swell that occurs at the start of the swell cycle and how this eventually flattens off into a more gradual swell. This also highlights the dangers that a one-size-fits-all approach can create, and different circumstances require a different range of elastomers.

\subsection{Low Temperature Swelling}

As explained above, diffusion gradients and mechanical elastomer characteristics interact to create the actual physical elastomer swelling. With low temperatures, diffusion is very low and slow and the elastomer base is less flexible, which limits performance dramatically. Absolute swell is also very low (this is the absolute amount an elastomer will swell at those 
conditions irrespective of how thick the swelling elastomer actually is. It will never swell more than the absolute swell because of the effects swell has on lowering the already low diffusion gradient). To create swell at lower temperatures, it is important that the diffusion gradient be improved and that the base elastomer mechanical characteristic be altered to utilize the available diffusion gradient better along with a balancing of the internal swelling pressure.

A new generation of oil swell elastomer is in the pipeline, which relies on the use of the new oilswelling SAP technology which is similar to those used in some of the low-end water swelling elastomers. These SAPs were initially developed to help oil spill clean-up but are now being applied to help to open up this lower temperature range oil swell requirement. They are proving to be an important technology in helping to improve the swell speed performance and when coupled to newly designed base elastomers are enabling swell speeds and swell levels to approach those experienced in oil swelling temperature ranges of more than $40^{\circ} \mathrm{C}$. These new commercial elastomer products are appearing and being used in low temperature fields where their effective working in real conditions has been demonstrated. Additionally, it means elastomer bases can be used that are not affected by the oil, as the bases are resistant to oil degradation. This has always been a limitation with oil swelling where only certain base elastomer families could be used as other base families would not react with the oil to actually swell. Now it is possible to use non-oil based swelling elastomer bases that are the same as those found in water swelling and are also not affected by their reaction with the motive fluids. This will dramatically improve their ultimate potential performance.

\subsection{High Temperature Elastomers}

The latest major developments have occurred at the higher end of the temperature range where demand is growing for elastomers well above $180^{\circ} \mathrm{C}$. What we see is that there is now a very distinct splitting of requirements for high temperature applications. For the likes of geothermal applications, swelling at this high temperature is more or less the requirement. However, where the requirement is for the use of steam, in for example tertiary recovery in oil wells, a lower temperature swell is required, but as the steam injection occurs, a resistance to these now much higher temperatures by the already swollen elastomer is vital. These are effectively two very different characteristics made all the more difficult by the probability of the steam well probably being cycled (stopping and starting) during steam injection.

As we move up to these and even higher temperatures levels, we hit two substantial walls. The base elastomer development takes considerably more time not just to develop, but also more importantly to test adequately. Probably even more important though is the development of bonding agents and systems suitable for use at these high temperatures. It is all well and good to have an elastomer that will work at these conditions but it has to stay stuck to the pipe and continue to hold a seal. However, the chemical bonding along with mechanical bonding is now proving to be as hard, if not harder to develop than the elastomer itself. This is especially so when one bears in mind that chemical bonding is very sensitive to base-pipe metallurgy and base elastomers. This applies to all elastomers whether used in high temperatures or not. Long-term bonding requires considerable knowledge and testing to achieve bonding on different substrates commensurate with the expected life expectancy of the seal. It is frequently overlooked, but it is the bonding that holds the swelling forces in the axial direction; the load that is applied to the bonding is much higher in a 12 -foot seal than in a 1-foot seal, even though the bonding strength remains the same. Efforts to restrict swelling at the end of a seal do this at the cost of the sealing capacity and often have little to do with any seal pressure extrusion.

This then, coupled with the type of system needed to both measure and test the elastomers, means a considerable step into a new world. While base elastomers are constantly being developed, the higher the temperature of the swelling elastomer, the deeper we enter into a totally new field of science with chemical reactions taking place between elastomer ingredients. At the same time, one can witness reactions between these ingredients and the swelling fluids (at temperatures and pressures), all of which influence the mechanical properties of the elastomer immediately and over an extended period of time. This means that not only do we need to test the elastomer as such for mechanical properties at elevated temperatures as well as later seal design versus pressures, but we also must create new tests and testing parameters to ensure that we understand what is being seen and measured. We must ensure that we are actually creating a test that gives meaningful and understandable results.

The design of the test for both accuracy and repeatability is crucial to the performance of the elastomer. Swell curves have to be structurally created which enable the performance envelope boundaries to be determined, as these boundaries change according to differences in salinity and temperature. Understanding the conditions and compensation factors required, ensures that the results are both meaningful and properly understood. It is not just enough to plunk a piece of rubber in a pot and then measure the swell. Things happen during swelling that require compensation in the measurements being made. Some of these are quite simple, such as the change in salini- 
ty of the sample water during swelling which is caused by the sweet water being extracted from the brine and entering the elastomer. This does, however, result in a salinity increase in the motive brine and, depending on the volume of the initial motive fluids, can affect the results dramatically. Likewise, the results can be affected by compensating for salinity changes between brines created at room temperature that are then heated and used at elevated temperatures. These and a few others are the factors that have to be understood and examined in detail when creating testing regimens.

It goes without saying that while the above is important for determining swell rates (swell versus time) and ultimate swell against time, this must also be borne in mind when we look to the design of full scale testing for pressure holding capacity. Swell is a one-toone factor; that is, the elastomer swells a unit volume by removing that equivalent unit volume from the motive fluid in which it exists. When designing the pressure testing facilities, especially for large elastomer seal volumes, that removal of large volumes of fluid from the system to achieve swell also happens. This means that the test facilities have to have this capacity in the fluid circuits and the pressure vessel to ensure that the elastomer swell is not being influenced by a change in motive fluid composition caused by the swell that is actually occurring. Failure to do this results in great inaccuracy in the amount of swell present and consequently in the sealing pressure tests results actually achieved.

For optimum effect we internally use 3-5 samples of standardized format bonded to steel plate samplesthe precise number depends heavily on the elastomer and application. Individually, in separate measurement pots, the samples are swollen over time in order to assess swelling. We facilitate this swelling for motive fluids from distilled water by making small salinity changes in steps, beginning with sweet water and adding brine until the solution achieves a saturated state. This is then repeated with new samples, increasing the sample temperature by $20^{\circ} \mathrm{C}$ from under the lower end of the swell range for that elastomer, until elastomer failure is achieved at a high end temperature. Thereby we determine the full operating envelope of that elastomer for the full range of salinity and temperatures. The results are then compared for repeatability and only when found to be repeatable within predetermined tolerance is the formulation under test then frozen and the results averaged to create the envelope used in our swell calculator.

Bonding results before and after swelling are compared to ensure that the swelling forces during swelling do not affect the bonding, as well as to ensure that the temperature and fluid have not affected the level of bonding achieved or caused any significant bonding aging. Standardized International Standards Organizations (ISO) tests are used for the bonding test measurements for each of the different substrates required.

\section{Remote Automated Tester}

As the operating temperatures increase to above $100^{\circ}$ Celsius for water swelling elastomers or above the oil flashpoint for oil swelling elastomers, the difficulties and dangers in measuring performance accurately increase immensely. Even things that are technically simple at lower temperatures can prove difficult when testing real performance at elevated temperatures and high pressure conditions. We have done considerable work to design and build an automated unit that can be used to measure swell and sealing in high pressure situations and temperatures up to $300^{\circ} \mathrm{C}$. The system is designed to be a hands-off system that allows for little operator intervention and measures swelling in real temperature and pressure situations. For the first time, it is allowing researchers to gain insight into how swelling at elevated conditions takes place. At the same time, sample dimensions have to be sufficiently large to be representative of working elastomers. If the sample dimensions are too small, the results cannot be up scaled and are not representative of larger seal size performances.

The ability to achieve and then repeat swell results, as well as to confirm accurately the amount of swell is vital. In the past, it was clearly evident that when lab samples were cooled and measured for swell, results, especially those ascertained at higher temperatures yielded higher error margins when compared to those collected in live measurement situations. Additionally, it was and is vital to eliminate operator variations. These variations may creep into results when using other methods both for time issues and for pressurerelated items and disallow accurate and repeatable swell rate curves to be generated.

The measurement system that has been created is quite a simple method for measuring swell against time. The system works with a set rubber thickness on the outside of a shaft in a swell chamber. The thickness is kept constant but the shaft diameter is varied, bringing the sample closer or further away from the cell wall. As the rubber swells, it will reach a point where it will contact the measuring cell wall. By causing the pressure under the seal to pulsate, and measuring the pressure above and below the test piece, it is possible to compare the pressure pulse time below the sample with the pressure pulse time above the sample. As the elastomer swells, a time delay occurs between pulses both in the lower and upper sections of the shaft. This delay is caused by fluid resistance between the upper and lower parts and is caused by the elastomer's contact with the test cell wall. Through automation of the testing system, the height of the pressure pulse changes as the time delay is met. In this way, first con- 
tact can be determined and, with a variable time element, the point of sealing can also be reliably determined. By varying the shaft diameter and keeping the rubber thickness constant, it is possible to set up and measure a percentage of swell diameter versus time in live conditions. By repeating this for different thicknesses, salinities and temperatures, it is possible to create swell curves for various conditions without the need for operator intervention as would have been necessary in the old way of doing these tests. Freeing up the compound development engineer enables a reduction in the cost of development. It also allows the engineers to develop and fine tune the compound.

With the different known swell curves, it is now possible to replace the swell curve samples with test samples and, using the time obtained by the swell curves, allow the samples to swell. Additionally, an automatic test cycle allows a pressure test to be conducted for two limits: 1) pressure to failure, where the pressure is increased in steps until the sample fails, and 2) extrusion degree, where an operating pressure is achieved and the sample maintained or cycled under pressure over an extended period of time, until either failure occurs or the test is stopped. Comprehension of both limits gives a much deeper understanding of the elastomer sealing performance over load change, time, and temperature.

This is because swelling is not sealing, sealing has to be created by understanding how swelling occurs. The ability to test automatically and to format data directly from the data logger is vital for the development of second generation seals where the shape can contribute more to the sealing than the swelling. This is doubly so when low and high temperature sealing is required and the seal mechanics and physical characteristics heavily influence potential sealing performance. It is often forgotten that swelling is 3-dimensional, which means the seal also gets longer as it swells. This length change puts huge demands upon the bonding, especially when very long seals are created.

This, together with some other proprietary measurement systems, have enabled us to develop a new generation of patent pending seals where seal-shape geometry has contributed to not only improved sealing performance but also reduced running hang up and debris interference in horizontal well open-hole liner systems.

The use of this system also enables engineers to test the effects of surface treatment on finished samples' swelling rates and performance. Data show a huge variation in initial swell-rate, which has been observed in samples deliberately made differently in order to ascertain the most ideal manufacturing process and the necessary quality requirements for production. Simple factors, such as surface grinding, can have a huge impact on swelling rate if they are done incorrectly or poorly.

What is clear is that this basic but necessary fundamental research proves that a soundly engineered compound, together with a rigorous and defined testing program, can ensure customer satisfaction. This is especially necessary in an industry where there are no testing standards or compound qualifications that would otherwise ensure a product based on sound testing and technology.

\section{The Classic Swelling Elastomer}

Work continues on measuring and developing classic swelling elastomers. These studies are aimed primarily at extending and improving their envelopes. However, major trends in the industry will soon start to move rapidly away from these rather basic elastomers. Extensive efforts are put to look into and research some of the more useful chemical tricks that are possible with elastomers and has patents pending on a number of its research project studies which have looked more deeply into the chemistry of elastomers and their swelling performance.

\subsection{Triggering and/or Controlling Swelling}

Classic swelling elastomers swell when they come into contact with their designated motive fluids. It is a given fact, and unless some error has been made in the design of the application, swelling is guaranteed to occur. This can, however, be a potential problem on complex liners and completion systems where swelling seals are included, and extra time is required for these completions to be run with their liners into the well. Whilst the elastomer types and dimensions are engineered for this, should anything untoward happen and cause a delay in achieving depth, the swelling seal can cause the liner to get stuck during the running in process. In the worst case, this potentially can lead to an inability to remove the liner from the well.

\subsection{Controlled Swell}

Depending on operational constraints, in most wells water-swelling elastomers can be slowed down if required by decreasing the water temperature and/or increasing the salinity by timely fluid circulation. However, the swelling can only be slowed and this approach must only be seen as a very last resort solution. With this concern in mind, we have looked into this as an integral part of our on-going retrievable swelling packer development program, which is especially timely considering the increased use of swelling 


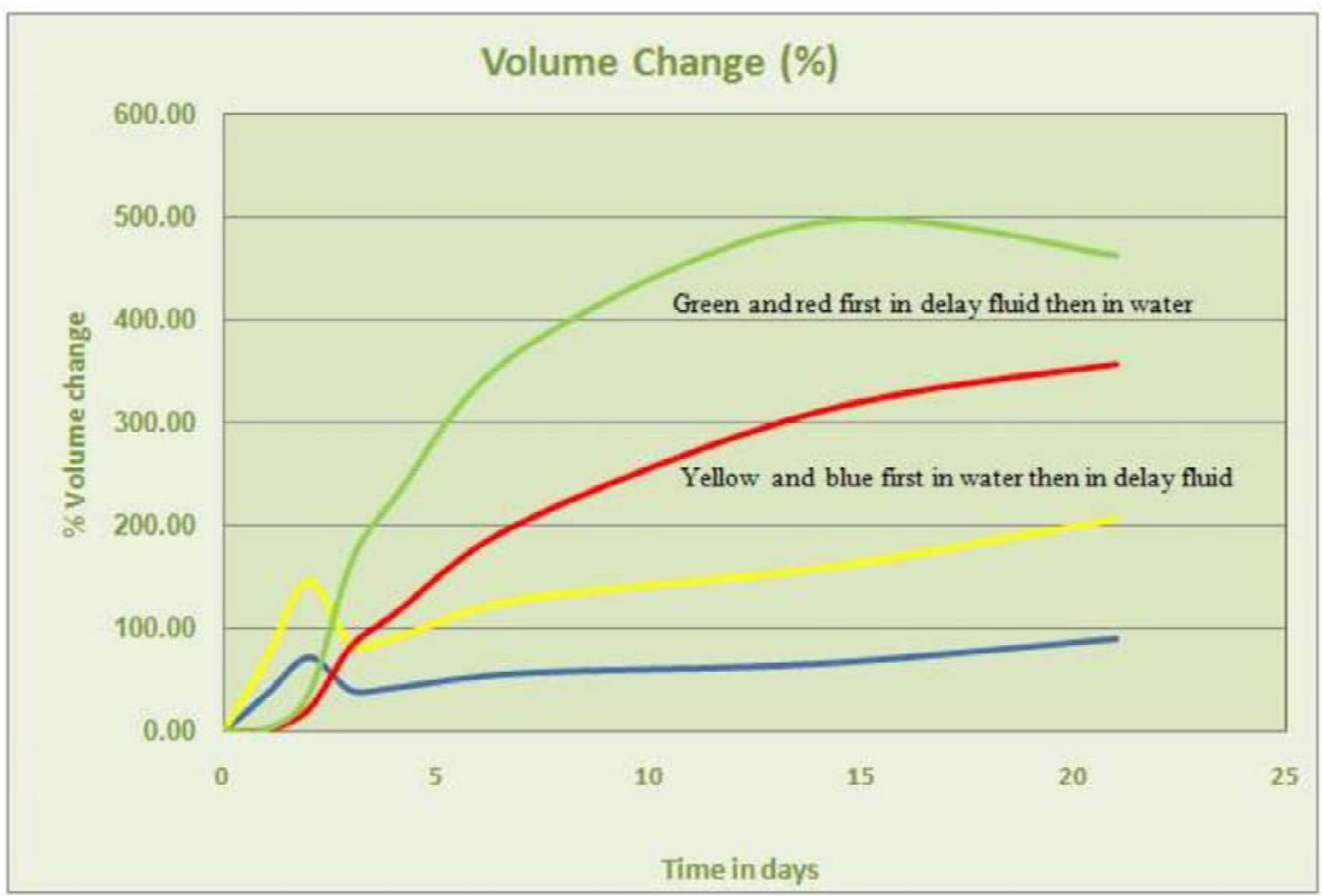

Figure 4. Elastomer swelling in water and in swell-delaying fluid

seals in open-hole situations. We have studied in great depth different ways of stopping, starting, and even, where possible, reversing swell, and now have a number of patents pending based upon our work here. We have researched and developed new, relatively simple, controllable techniques to stop and start water swelling on demand. This now gives us the ability to actually delay the onset of swelling where desired until it is required. This in turn means that we are able to increase elastomer dimensions, ensuring a commensurate reduction in swell time to first contact and, ultimately, to full seal. Whilst the development of a retrievable swelling packer is still some way off, this spin-off from the study is a valuable tool for the future.

Figure 4 shows the results of a simple test where four identical samples have been placed in fluid. The yellow and blue samples were placed first in distilled water for two days and then transferred to water containing a swell- delaying fluid. The green and red samples were placed in the water with the swell-delaying fluid and then transferred to distilled water. Clearly the swell-delaying fluid had an effect on both sets of samples.

\subsection{Triggered Swell}

We have developed a second and very exciting new technique that almost indefinitely delays swell. This technique is able to delay the occurrence of swell and then trigger it on demand to start whenever it may be desired or required do so. Although this technology is still in the experimental, laboratory stages, it will ulti- mately lead to a third generation of new elastomer product groups with what should be vastly enhanced abilities.

Figures 5 and 6 illustrate the degrees of volume swell being achieved using newly developed triggered swell elastomer/fluid combinations (note this is not dimensional swell, which is roughly $1 / 3$ of volume swell). The first shows a slight swell without triggering fluid addition, but at a low volume. These are the inherent swelling characteristics caused by the ingredients in the base elastomer. The effects of the triggering fluids can be clearly seen. Additionally, the swell control that the percentage of trigger fluid can be engineered to create can be seen. In the above graph, the effect that temperature has on a fixed swell trigger fluid percentage can also be seen. By managing the trigger fluid, it is possible to totally alter the swell curves to create a system-managed swell to suit the applications required.

These new offerings, together with probable developments by others in the future, will amount to a potential second-step change in the way wells are built. This step is as large as if not larger than the one that which was created by the pioneering, introduction, and application of water swelling elastomers almost 10 years ago.

\section{The Way Forward}

Elastomers, and elastomer and chemical engineering are not really areas that have received the focus 


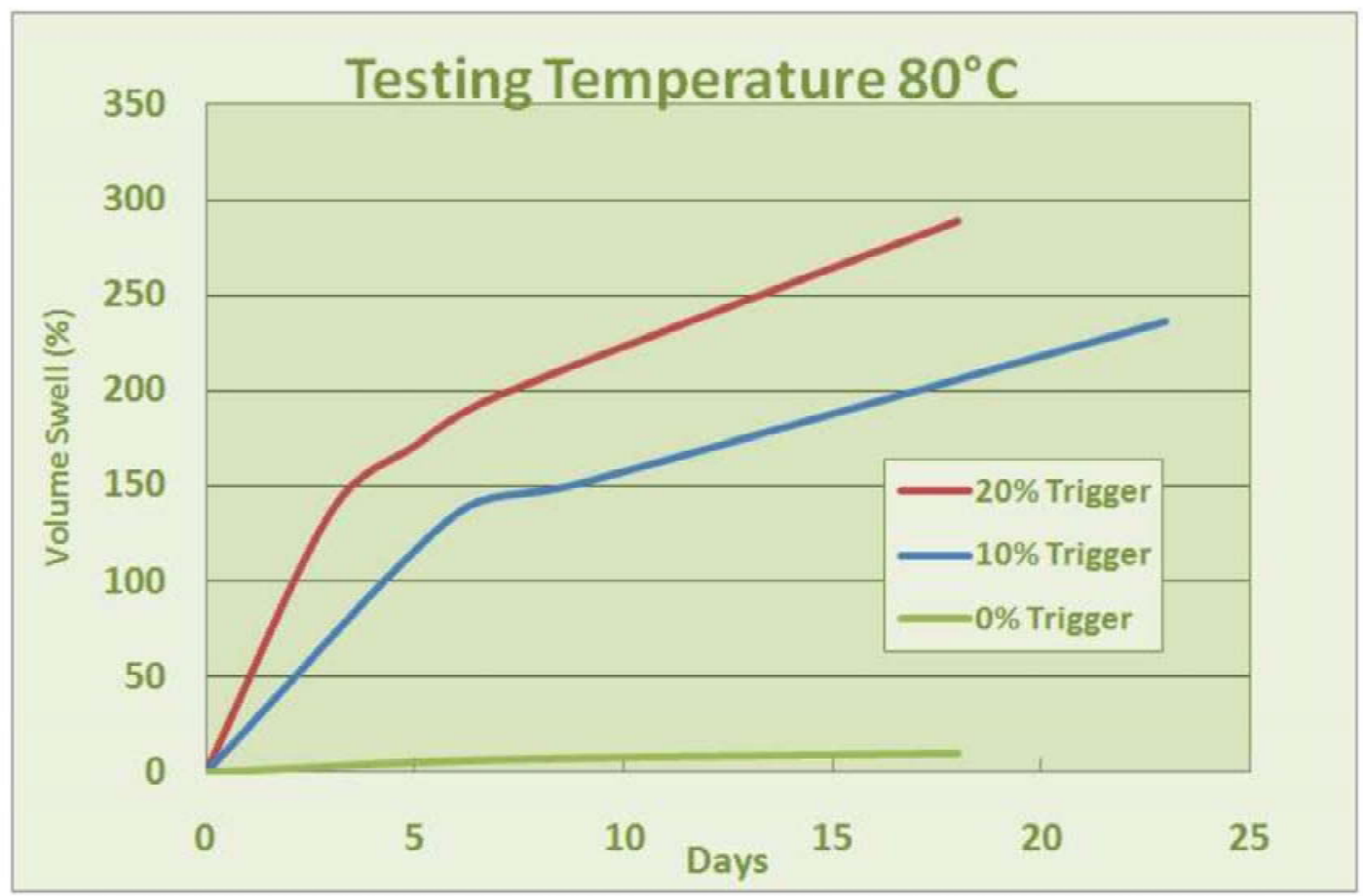

Figure 5. Effect of triggering amount on volume swelling

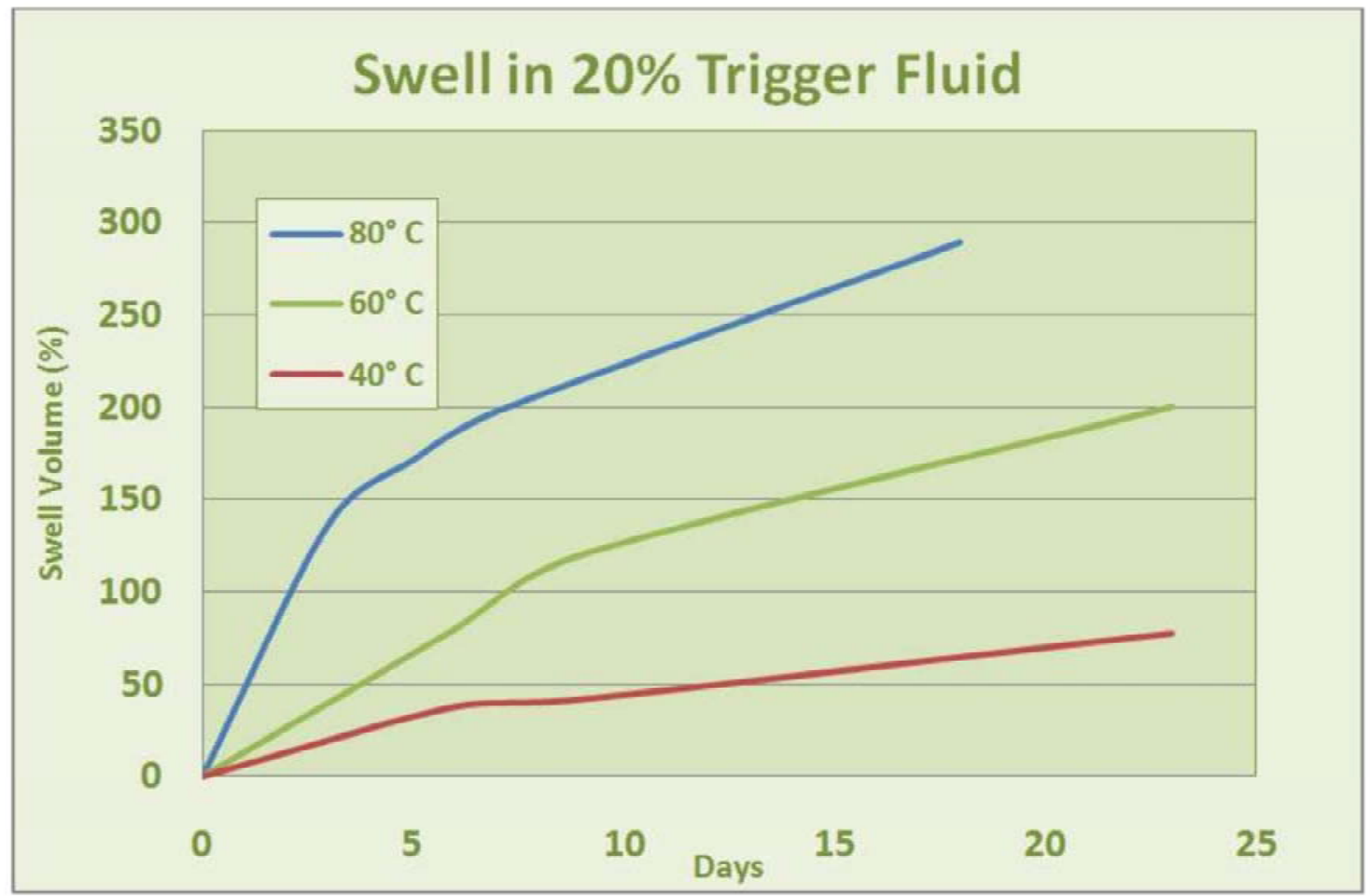

Figure 6. Effect of temperature on volume swelling for $20 \%$ trigger

and attention in well design that they deserve. Whilst most well engineers have a sound and deep understanding of metallurgy as well as well mechanics, they do tend to have a very poor to almost non-existent understanding of the potential that elastomers and elastomer chemistry can bring to well engineering.
Most of them only have had contact with elastomers via packer elements that utilize what is possibly the weakest of the characteristics of elastomers.

What is clear is the potential that chemical engineering offers to mechanical engineers in their quest for ever simpler and more cost effective sealing in 
wells. This technology is only beginning to be understood and used. Elastomer engineers working with mechanical equipment designers and well engineers can alter the face of well design and building very substantially, as can be seen by the impact seen of swelling seals on shale gas and oil recovery. Hard wearing elastomers can potentially replace hard facing on drilling equipment. They can create hard wearing and cost effective corrosion layers on equipment prone to corrosion attack, at a cost substantially lower than that associated with exotic metallurgy.

What is clear is that well engineers do not know enough about elastomers, and elastomer engineers do not know enough about well design to automatically create solutions to problems. There is clearly a large deal of education that needs to be given by elastomer engineering companies on both swelling and nonswelling elastomers and their potential uses to completion equipment designers and equipment users. Simultaneously, the completion equipment designers and users clearly need to educate the elastomer companies on their potential requirements, wishes, and dreams. There is still a real disconnect between both parties as to what they can mean to each other and the future market and trends that can offer joint options and commercial as well as technical possibilities. This disconnectedness needs to be addressed by all parties to help the future of cost effective hydrocarbon production operations make a move into the future. It was, after all, elastomer engineers who created the solution that has freed up shale gas and oil, while it was the well engineers who saw the potential and grasped it.

\section{References}

Anjum MG, Shafeeq U, Younas MA (2010), Solid expandable tubular combined with swellable elastomers facilitate muli-zonal isolation and fracturing. SPE Paper \# 131489-MS, International Oil and Gas Conference and Exhibition, Beijing, China.

M2 Polymer Technologies Incorporated. (2012), How SAP works. From: www.m2polymer.com/ html/ chemistry_sap.html.

Pal K, Rajasekar R, Kang DJ, Zhang ZX, Pal SK, Das CK, Kim JK (2010), Effect of fillers on natural rubber/high styrene rubber blends with nano silica: Morphology and wear. Materials and Design 31(2):677-686.

Wack H, Hintemann D, Michael H, Buschner N (2011), Preparation and properties of swellable thermoplastic elastomer alloys based on elastomeric powder, polypropylene, and superabsorbent polymer. Journal of Applied Polymer Science 120(3):1290-1296.

Wikipedia (2012), Fick's first and second laws. From http://en.wikipedia.org/wiki/Fick\%27s_law.

Young D, Sevre AK, Smith PE, al-Madlouh M, Awang MZ (2011), Effects of rapid gas decompression on swellable rubber and common oilfield rubber compounds. SPE paper \# 149076-MS, SPE/DGS Saudi Arabia Section Technical Symposium and Exhibition, Al-Khobar, Saudi Arabia. 


\section{Appendix}

\section{Appendix - A1}

Fick's first law relates the diffusive flux to the concentration under the assumption of steady state. It postulates that the flux goes from regions of high concentration to regions of low concentration, with a magnitude that is proportional to the concentration gradient (spatial derivative). In one spatial dimension, the law is

$$
J=-D \frac{\partial \phi}{\partial x}
$$

where $J$ is the diffusion flux (amount of substance) per unit area per unit time $\left(\mathrm{mol} / \mathrm{m}^{2}-\mathrm{s}\right), D$ is the diffusion coefficient (diffusivity) $\left(\mathrm{m}^{2} / \mathrm{s}\right), \Phi$ is the concentration for ideal mixtures $\left(\mathrm{mol} / \mathrm{m}^{3}\right)$, and $x$ is the position $(\mathrm{m})$. The diffusion coefficient is proportional to the squared velocity of the diffusing particles, which depends on the temperature and viscosity of the fluid, and the size of the particles according to the Stokes-Einstein relation. In dilute aqueous solutions, the diffusion coefficients of most ions are similar and have values that at room temperature are in the range of $0.6 \times 10^{-9}$ to $2 \times 10^{-9} \mathrm{~m}^{2} / \mathrm{s}$. For biological molecules, the diffusion coefficients normally range from $10^{-11}$ to $10^{-10} \mathrm{~m}^{2} / \mathrm{s}$. In two or more dimensions we must use $\nabla$, the del or gradient operator, which generalizes the first derivative, obtaining

$$
J=-D \nabla \phi
$$

The driving force for the one-dimensional diffusion is represented by the quantity $(\delta \Phi / \delta x)$, which for ideal mixtures is the concentration gradient. In chemical systems other than the ideal solutions or mixtures, the driving force for diffusion of each species is the gradient of chemical potential of this species. Hence, Fick's first law for 1-D case can be written as

$$
J_{i}=-\frac{D c_{i}}{R T} \frac{\partial \mu_{i}}{\partial x}
$$

where the subscript ' $\mathrm{i}$ ' indexes the ith species, $c$ is the concentration $\left(\mathrm{mol} / \mathrm{m}^{3}\right), R$ is the universal gas constant $\left(\mathrm{J} /\left({ }^{\circ} \mathrm{K} \mathrm{mol}\right)\right), T$ is the absolute temperature $\left({ }^{\circ} \mathrm{K}\right)$, and $\mu$ is the chemical potential $(\mathrm{J} / \mathrm{mol})$. If the primary variable is mass fraction $\left(y_{\mathrm{i}}\right)$ then the above equation changes to

$$
J_{i}=-\rho D \nabla y_{i}
$$

where $\rho$ is the fluid density. Note that the density is outside the gradient operator.

\section{Appendix - A2}

Fick's second law predicts how diffu sion causes the concentration to change with time:

$$
\frac{\partial \phi}{\partial t}=D \frac{\partial^{2} \phi}{\partial x^{2}}
$$


The following can be derived from Fick's first law and the mass conservation in the absence of any chemical reactions:

$$
\frac{\partial \phi}{\partial t}+\frac{\partial}{\partial x} J=0 \Rightarrow \frac{\partial \phi}{\partial t}-\frac{\partial}{\partial x}\left(D \frac{\partial}{\partial x} \phi\right)=0
$$

Assuming the diffusion coefficient $D$ to be a constant, we can exchange the orders of the differentiation and multiply by the constant

$$
\frac{\partial}{\partial x}\left(D \frac{\partial}{\partial x} \phi\right)=D \frac{\partial}{\partial x} \frac{\partial}{\partial x} \phi=D \frac{\partial^{2} \phi}{\partial x^{2}}
$$

and, thus, receive the form of the Fick's equations as was stated above. For the case of diffusion in two or more dimensions, Fick's second law becomes

$$
\frac{\partial \phi}{\partial t}=D \nabla^{2} \phi
$$

which is analogous to the heat equation. If the diffusion coefficient is not a constant, but depends upon the coordinate and/or concentration, Fick's second law yields

$$
\frac{\partial \phi}{\partial t}=D *(D \nabla \phi)
$$

An important example is the case where $\Phi$ is at a steady state (ie. the concentration does not change over time) so that the left part of the above equation is identically zero. In one dimension with constant $D$, the solution for the concentration will be a linear change of concentrations along $x$. In two or more dimensions we obtain

$$
\nabla^{2} \phi=0
$$

which is Laplace's equation, the solutions, which are called harmonic functions by mathematicians.

\section{APPENDIX - A3}

How does SAP work? Acrylate polymers when in a dry state resemble curled up curly-Qs or pigs tails. 


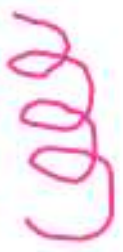

When these molecules are placed in water, hydrogen bonding with the $\mathrm{HOH}$ surrounding them causes them to unfold and straighten out so that they resemble strands of spaghetti.
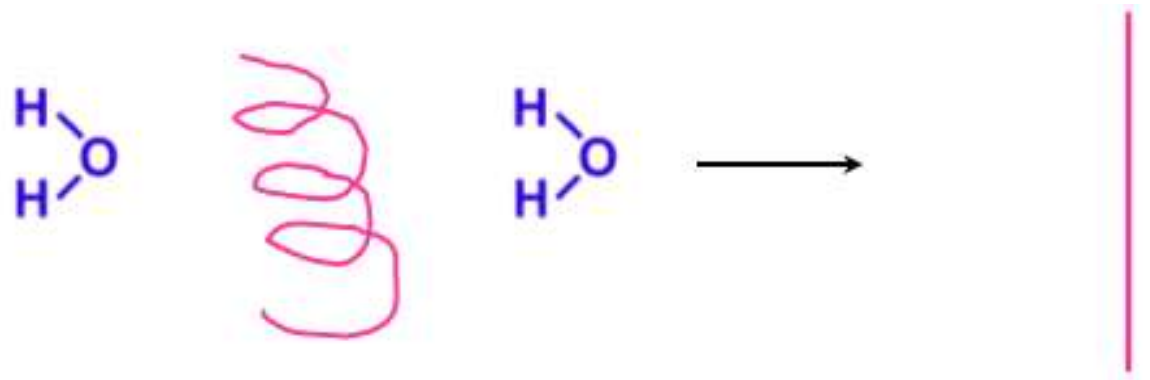

When the molecules straighten out, they increase the viscosity of the surrounding liquid. That is why several types of acrylates are used as thickeners. Super absorbent chemistry requires two things: the addition of small cross-linking molecules between the polymer strands, and the partial neutralization of the carboxyl acid groups $(-\mathrm{COOH})$ along the polymer backbone $(-\mathrm{COO}-\mathrm{Na}+)$. Water molecules are drawn into the network across a diffusion gradient which is formed by the sodium neutralization of the polymer backbone. The polymer chains want to straighten but are constrained due to the cross-linking. Thus, the particles expand as water moves into the network.

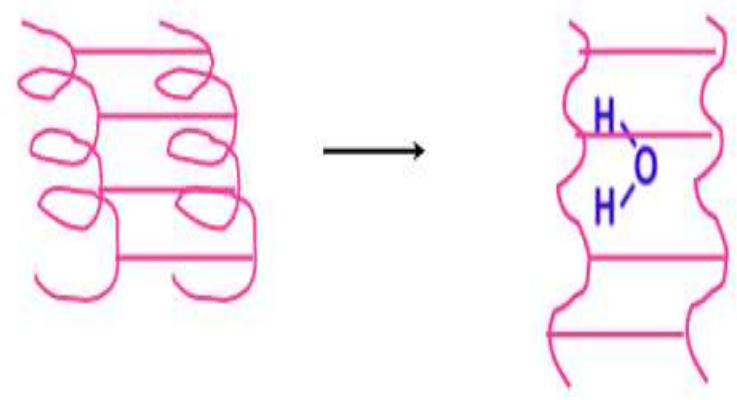

The water is tightly held in the network by hydrogen bonding. Many soluble metals will also ionexchange with the sodium along the polymer backbone and be bound. Here is what a super absorbent molecule looks like in greater detail: 


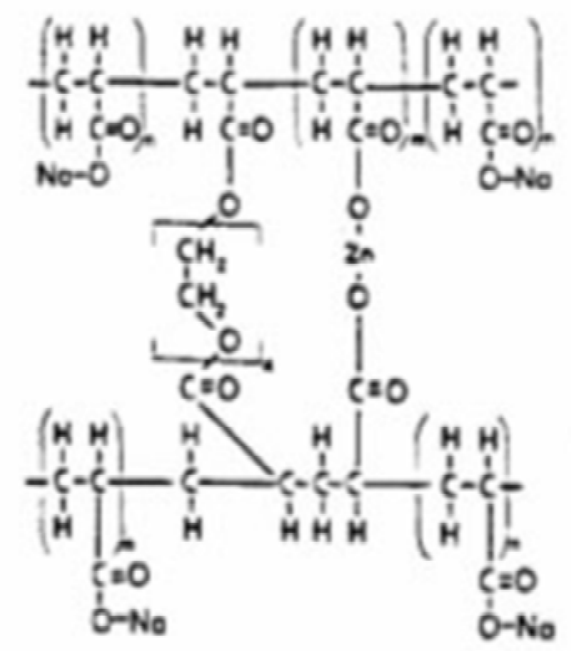

And here's what a super absorbent network looks like on a larger scale.

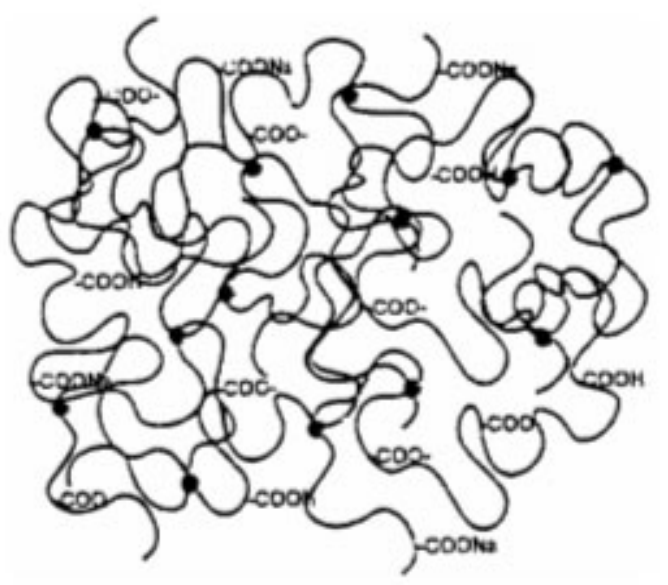

